\title{
A PRINCIPLE OF LINEARIZED STABILITY FOR NONLINEAR EVOLUTION EQUATIONS
}

\author{
NOBUYUKI KATO
}

\begin{abstract}
We present a principle of linearized stability of stationary solutions to nonlinear evolution equations in Banach spaces. The well-known semilinear case is shown to fit into our framework. Applications to nonlinear population dynamics and to functional differential equations are also considered.
\end{abstract}

\section{INTRODUCTION}

It is well known as a principle of linearized stability that stability properties of stationary solutions $\bar{u}$ of ordinary differential equations $d u / d t=F(u)$ in $\mathbb{R}^{n}$ are determined by the eigenvalues of the differential $D F(\bar{u})$ of $F$ at $\bar{u}$. Similar facts are also known for semilinear evolution equations $d u / d t=L u+$ $F(u)$ in Banach spaces and many applications to partial differential equations of semilinear type has been investigated by many authors. See for example $[16$, Chapter 11], [8], [17, Chapter 4]. form

In this paper, we are interested in fully nonlinear evolution equations of the

$$
\frac{d}{d t} u(t)+A u(t)=0, \quad t \geq 0,
$$

where $A$ is a quasi- $m$-accretive operator in a Banach space. Our first purpose is to formulate a principle of linearized stability for the above nonlinear equations and establish the local asymptotic stability result. In general, $A$ is not even continuous and so it is a matter of course that the Fréchet derivative of $A$ cannot be considered. Thus we encounter the problem how we should define a linearized operator for $A$. We will adopt the idea of tangent cones from 'nonsmooth analysis'. Especially the notion of proto-differentiability introduced recently by Rockafellar [14] is found to be appropriate for our purpose. The abstract semilinear equations are shown to fit into our framework as shown in Corollary 2.2.

Our second objective is to show how our principle can be applied to agedependent population dynamics and functional differential equations in Banach

Received by the editors December 13, 1993.

1991 Mathematics Subject Classification. Primary 47H20, 47H15, 47H06; Secondary 35B35, 92D25, 34K20.

Key words and phrases. Linearized stability, $m$-accretive operators, proto-derivatives, nonlinear evolution equations, population dynamics, functional differential equations.

This research was supported by the Grant-in-Aid for scientific research from the Ministry of Education of Japan. 
spaces. As developed in [17], the well-known principle of semilinear case is applicable only to the population dynamics of semilinear type and the different methods are needed to treat so-called fully nonlinear population dynamics. See also [13]. In view of this, our principle has advantage of applying directly to nonlinear population dynamics. We should remark that Desch and Schappacher [4] have obtained a principle of linearized stability focused on nonlinear semigroups rather on nonlinear equations themselves and that their results also have applications to the same problem presented here as shown recently by Grabosch [6] and Parrott [11]. Other related results have been obtained by Greiner [7], in which abstract evolution equations with semilinear boundary conditions have been considered. The equations are more general than our model of population dynamics.

We will prepare some notations and preliminary lemmas in $\S 1$. In $\S 2$, we will give principles of linearized stability for nonlinear and semilinear equations (Theorem 2.1 and Corollary 2.2). Their proofs are done in $\S 4$, and $\S 3$ is devoted to analyze the regularized equation and its linearization. We will discuss the applications to age-dependent population dynamics in $\S 5$ and functional differential equations in Banach spaces in $\S 6$.

\section{Preliminaries}

In order to formulate our results, we need some notions from nonsmooth analysis. Let $X$ and $Y$ be Banach spaces and let $F: X \rightarrow 2^{Y}$ be a multi-valued operator, which domain, range, and graph are defined as follows: $\mathscr{D}(F):=\{x \in$ $X \mid F(x) \neq \varnothing\}, \mathscr{R}(F):=\bigcup_{x \in \mathscr{D}(F)} F(x)$, and $\mathscr{G}(F):=\{(x, y) \in X \times Y \mid x \in$ $\mathscr{D}(F)$ and $y \in F(x)\}$. For $(x, y) \in \mathscr{G}(F)$, we define multi-valued operators $\partial_{i} F(x, y), \partial_{s} F(x, y): X \rightarrow 2^{Y}$ by

$$
\begin{aligned}
& \mathscr{G}\left(\partial_{i} F(x, y)\right)=\liminf _{t \downarrow 0} t^{-1}[\mathscr{G}(F)-(x, y)], \\
& \mathscr{G}\left(\partial_{S} F(x, y)\right)=\limsup _{t \downarrow 0} t^{-1}[\mathscr{G}(F)-(x, y)],
\end{aligned}
$$

where $\liminf \operatorname{in}_{t \downarrow 0}$ and limsup $\sup _{t \downarrow 0}$ are taken in the sense of set convergence (cf. [1]). In other words,

$$
\begin{aligned}
& (u, v) \in \mathscr{G}\left(\partial_{i} F(x, y)\right) \Longleftrightarrow \forall t_{n} \downarrow 0 \exists\left(u_{n}, v_{n}\right) \rightarrow(u, v) \text { in } X \times Y: \\
& \left(x+t_{n} u_{n}, y+t_{n} v_{n}\right) \in \mathscr{G}(F) \text {, } \\
& (u, v) \in \mathscr{G}\left(\partial_{s} F(x, y)\right) \Longleftrightarrow \exists t_{n} \downarrow 0 \exists\left(u_{n}, v_{n}\right) \rightarrow(u, v) \text { in } X \times Y: \\
& \left(x+t_{n} u_{n}, y+t_{n} v_{n}\right) \in \mathscr{G}(F) \text {. }
\end{aligned}
$$

The operator $\partial_{s} F(x, y)$ is called the contingent derivative, while $\partial_{i} F(x, y)$ is called the intermediate derivative by Frankowska [5] because it lies between the contingent derivative and the Clarke tangent derivative. It is apparent that $\partial_{i} F(x, y) \subset \partial_{s} F(x, y)$ in the sense of graph inclusion. If $\partial_{i} F(x, y)=$ $\partial_{s} F(x, y)$, we denote it by $\partial F(x, y)$ and $F$ is said to be proto-differentiable at $x$ relative to $y$ and $\partial F(x, y)$ is called the proto-derivative of $F$ at $x$ relative to $y$ by Rockafellar [14]. When $F$ is single-valued, we write $\partial F(x):=$ $\partial F(x, F(x))$ and call it the proto-derivative of $F$ at $x$.

Let $F$ be a single-valued operator from $X$ into $Y$ with open domain $\mathscr{D}(F)$. We say that $F$ is Gâteaux differentiable at $x \in \mathscr{D}(F)$ if there exists a $d F(x) \in$ 
$\mathscr{L}(X, Y)$ ( $\equiv$ the space of all bounded linear operators from $X$ into $Y$ ) such that

$$
(Y-) \lim _{t \downarrow 0} t^{-1}[F(x+t h)-F(x)]=d F(x) h \quad \text { for all } h \in X .
$$

The operator $d F(x)$ is called the Gâteaux derivative of $F$ at $x$.

Lemma 1.1. Let $F: X \rightarrow Y$ be a single-valued Lipschitz continuous mapping with $\mathscr{D}(F)=X$. If $\partial_{i} F(x) \in \mathscr{L}(X, Y)$, then $F$ is Gâteaux differentiable at $x$ and $\partial_{i} F(x)=d F(x)$.

Proof. Let $h \in X$. Since $\left(h, \partial_{i} F(x) h\right) \in \mathscr{G}\left(\partial_{i} F(x)\right)$, for any $t_{n} \downarrow 0$, there exists a sequence $\left(h_{n}, k_{n}\right)$ such that $\left(h_{n}, k_{n}\right) \rightarrow\left(h, \partial_{i} F(x) h\right)$ in $X \times Y$ and $(x+$ $\left.t_{n} h_{n}, F\left(x+t_{n} k_{n}\right)\right) \in \mathscr{G}(F)$. Hence $t_{n}^{-1}\left[F\left(x+t_{n} h_{n}\right)-F(x)\right]=k_{n} \rightarrow \partial_{i} F(x) h$. Since $F$ is Lipschitzian, we have $t_{n}^{-1}\left[F\left(x+t_{n} h\right)-F(x)\right] \rightarrow \partial_{i} F(x) h$.

As a more restricted concept than Gâteaux differentiability, we will use the Fréchet differentiability. The following definition relies on [17, Definition 2.4, p.63]. Here we denote the norms of $X, Y$, and $\mathscr{L}(X, Y)$ by $|\cdot|_{X},|\cdot|_{Y}$, and $\|\cdot\|_{\mathscr{L}(X, Y)}$, respectivly.

Definition 1.1. A single-valued operator $F: \mathscr{D}(F) \subset X \rightarrow Y$ is said to be Fréchet differentiable at $x \in \mathscr{D}(F)$ if there exists a $D F(x) \in \mathscr{L}(X, Y)$ such that

$$
F(y)=F(x)+D F(x)(y-x)+o(y-x), \quad \forall y \in \mathscr{D}(F),
$$

where $o$ is a function from $X$ into $Y$ satisfying $|o(z)|_{Y} \leq b(r)|z|_{X}$ for all $z \in$ $X$ with $|z|_{X} \leq r$ for some continuous increasing function $b:[0, \infty) \rightarrow[0, \infty)$ such that $b(0)=0$. Further, $F$ is said to be continuously Fréchet differentiable on $\mathscr{D}(F)$ provided that $F$ is Fréchet differentiable at each $x \in \mathscr{D}(F)$ and there exists a continuous increasing function $d:[0, \infty) \rightarrow[0, \infty)$ such that

$$
\left\|D F\left(x_{1}\right)-D F\left(x_{2}\right)\right\|_{\mathscr{L}(X, Y)} \leq d(r)\left|x_{1}-x_{2}\right|_{X}, \quad \forall x_{i} \in \mathscr{D}(F), \quad\left|x_{i}\right|_{X} \leq r
$$

$$
(i=1,2) \text {. }
$$

Remark 1.1. Let $F: X \rightarrow Y$ be continuously Fréchet differentiable on $X$. Then $F$ is locally Lipschitz continuous in the sense that there exists an increasing function $c:[0, \infty) \rightarrow[0, \infty)$ such that

$$
|F(z)-F(x)|_{Y} \leq c(r)|z-x|_{X} \quad \forall z, x \in X,|z|_{X} \leq r,|x|_{X} \leq r .
$$

Indeed, we can take $c(r)=d(r) r+\|D F(0)\|_{\mathscr{L}(X, Y)}$. For $r>0$, we define the radial truncation of $F$ by

$$
F_{r}(\phi):= \begin{cases}F(\phi) & \text { if }|\phi|_{X} \leq r, \\ F\left(r \phi /|\phi|_{X}\right) & \text { if }|\phi|_{X}>r .\end{cases}
$$

As shown in [17, Proposition 3.10, p.95], $F_{r}$ is globally Lipschitz continuous with constant $\left\|F_{r}\right\|_{\text {Lip }}=2 c(r)$. Moreover, $F_{r}$ is continuously Fréchet differentiable on the open ball $B_{r}(0)$ of radius $r$ centered at 0 in $X$, more precisely, $F_{r}(\phi+h)=F_{r}(\phi)+D F(\phi) h+o(h)$ for $|\phi|_{X}<r,|\phi+h|_{X}<r$; and $\|D F(\phi)-D F(\psi)\|_{\mathscr{L}(X, Y)} \leq d(s)|\phi-\psi|_{X}$ for $|\phi|_{X},|\psi|_{X} \leq s<r$.

Let $(X,|\cdot|)$ be a Banach space. A possibly multi-valued operator $Q: X \rightarrow$ $2^{X}$ is said to be accretive if

$$
|x-\hat{x}+\lambda(y-\hat{y})| \geq|x-\hat{x}| \quad \text { for all }(x, y), \quad(\hat{x}, \hat{y}) \in \mathscr{G}(Q) \text {, and } \lambda>0 \text {. }
$$


An accretive operator $Q$ is called $m$-accretive if the range condition $\mathscr{R}(I+\lambda Q)=X$ is satisfied for all (equivalently, for some) $\lambda>0$, where $I$ is the identity operator. If $Q+\omega I$ is $m$-accretive for some $\omega \in \mathbb{R}$, we define the resolvent and the Yosida approximation by

$$
J_{\lambda}^{Q}:=(I+\lambda Q)^{-1} \text { and } Q_{\lambda}:=(1 / \lambda)\left(I-J_{\lambda}^{Q}\right) \text { for } \lambda>0 \text { with } \lambda \omega<1,
$$

respectively. It is easily seen that $Q+\omega I$ is $m$-accretive if and only if $J_{\lambda}^{Q}$ is a single-valued operator defined on all of $X$ and satisfies $\left|J_{\lambda}^{Q} x-J_{\lambda}^{Q} y\right| \leq$ $(1-\lambda \omega)^{-1}|x-y|$ for $x, y \in X$. The Yosida approximation $Q_{\lambda}$ is a Lipschitz mapping with Lipschitz constant $(2-\lambda \omega) / \lambda(1-\lambda \omega)$ and $Q_{\lambda}+\omega(1-\lambda \omega)^{-1}$ is $m$-accretive. In addition, $J_{\lambda}^{Q+\omega I} x=J_{\lambda(1+\lambda \omega)^{-1}}^{Q}\left((1+\lambda \omega)^{-1} x\right)$.

Lemma 1.2. Let $Q+\omega I$ be an $m$-accretive operator for some $\omega \in \mathbb{R}$. Suppose that $\partial_{i} Q(x, y)+\omega I$ is $m$-accretive for $(x, y) \in \mathscr{G}(Q)$. Then $\left(\partial_{i} Q(x, y)\right)_{\lambda}=$ $\partial_{i} Q_{\lambda}(x+\lambda y, y)$. The same fact is also true if $\partial_{i}$ is replaced by $\partial_{s}$.

Proof. One easily checks that $(u, v) \in \mathscr{G}\left(\left(\partial_{i} Q(x, y)\right)_{\lambda}\right)$ is equivalent to the fact that for any $t_{n} \downarrow 0$, there exists a sequence $\left(w_{n}, z_{n}\right) \rightarrow(u-\lambda v, v)$ such that $\left(x+t_{n} w_{n}, y+t_{n} z_{n}\right) \in \mathscr{G}(Q)$. On the other hand, $(u, v) \in \mathscr{G}\left(\partial_{i} Q_{\lambda}(x+\lambda y, y)\right)$ is equivalent to the fact that for any $t_{n} \downarrow 0$, there exists a sequence $\left(u_{n}, v_{n}\right) \rightarrow$ $(u, v)$ such that $\left(x+t_{n}\left(u_{n}-\lambda v_{n}\right), y+t_{n} v_{n}\right) \in \mathscr{G}(Q)$. Thus to prove the inclusion $\left(\partial_{i} Q(x, y)\right)_{\lambda} \subset \partial_{i} Q_{\lambda}(x+\lambda y, y)$, we put $u_{n}=w_{n}+\lambda z_{n}$ and $v_{n}=z_{n}$. To prove the converse inclusion, we put $w_{n}=u_{n}-\lambda v_{n}$ and $z_{n}=v_{n}$. The case $\partial_{s}$ is similar.

\section{LINEARIZED STABILITY}

Let $(X,|\cdot|)$ be a Banach space and $A: \mathscr{D}(A) \subset X \rightarrow X$ be a single-valued operator such that $A+\omega I$ is $m$-accretive for some $\omega \geq 0$. In this section, we deal with the nonlinear evolution equation

$$
\frac{d}{d t} u(t)+A u(t)=0, \quad t \geq 0
$$

We will give a principle of linearized stability which ensures the asymptotic stability of stationary solutions of (E). It is well known that $A$ generates a strongly continuous nonlinear semigroup $\{S(t)\}_{t \geq 0}$ on $\overline{\mathscr{D}(A)}$ satisfying

$$
|S(t) x-S(t) y| \leq e^{\omega t}|x-y|, \quad \forall x, y \in \overline{\mathscr{D}(A)}
$$

by the Crandall-Liggett theorem. Putting $u(t):=S(t) u_{0}, u_{0} \in \overline{\mathscr{D}(A)}$, we think that $u \in C([0, \infty) ; \overline{\mathscr{D}(A)})$ gives a unique 'generalized' solution of the Cauchy problem (E) with initial data $u_{0}$.

Definition 2.1. $\bar{u} \in \mathscr{D}(A)$ is said to be a stationary solution of (E) if $A \bar{u}=0$, or equivalently, $S(\bar{t}) \bar{u}=\bar{u}$.

Throughout this section, we fix a stationary solution $\bar{u}$ of (E) and assume the following hypotheses:

(H1) There exists an open ball $B_{r}(\bar{u})$ of radius $r>0$ with center $\bar{u}$ such that for each $x \in B_{r}(\bar{u}) \cap \mathscr{D}(A)$, there exists a single-valued linear operator $\partial A(x): \mathscr{D}(\partial A(x)) \subset X \rightarrow X$ such that $\partial A(x)+\omega I$ is $m$-accretive and

$$
\mathscr{G}(\partial A(x))=\lim _{t \downarrow 0} t^{-1}[\mathscr{G}(A)-(x, A x)] .
$$


(H2) There exist a $\lambda_{\bar{u}}>0$ and a nondecreasing function $L_{\bar{u}}:[0, \infty) \rightarrow$ $[0, \infty)$ such that

$$
\left|J_{\lambda}^{\partial A(x)} v-J_{\lambda}^{\partial A(z)} v\right| \leq \lambda|x-z| L_{\bar{u}}(|v|)
$$

for $0<\lambda<\lambda_{\bar{u}}, x, z \in B_{r}(\bar{u}) \cap \mathscr{D}(A)$, and $v \in X$.

(H1) states that $A$ is proto-differentiable on $B_{r}(\bar{u}) \cap \mathscr{D}(A)$ and the protoderivative $\partial A(x)$ is single-valued and $\partial A(x)+\omega I$ is $m$-accretive. (H2) is a condition which describes the dependence of $\partial A(x)$ on the variable $x$. Let $\mathscr{L}(X):=\mathscr{L}(X, X)$ and denote its operator norm by $\|\cdot\|$. For a $\left(C_{0}\right)$ semigroup $\{T(t)\}$ with infinitesimal generator $Q$, the growth bound of $\{T(t)\}$ or the type of $Q$ is defined by

$$
\omega_{0}(Q):=\lim _{t \rightarrow \infty} t^{-1} \log \|T(t)\| .
$$

Our main result is stated as follows:

Theorem 2.1. Let $\bar{u}$ be a stationary solution of $(\mathrm{E})$ and assume the above hypotheses $(\mathrm{H} 1)$ and $(\mathrm{H} 2)$. If the proto-derivative $-\partial A(\bar{u})$ is the infinitesimal generator of a $\left(C_{0}\right)$-semigroup and $\omega_{0}(-\partial A(\bar{u}))<0$, then $\bar{u}$ is exponentially asymptotically stable in the sense that there exist constants $\eta>0, C \geq 1, \alpha>0$ such that

$$
u_{0} \in \overline{\mathscr{D}(A)}, \quad\left|u_{0}-\bar{u}\right|<\eta \Rightarrow\left|S(t) u_{0}-\bar{u}\right| \leq C e^{-\alpha t}\left|u_{0}-\bar{u}\right|, \quad \forall t \geq 0 .
$$

Next we consider the following semilinear equation:

$$
\frac{d}{d t} u(t)=(L+F) u(t),
$$

where $L: \mathscr{D}(L) \subset X \rightarrow X$ is the infinitesimal generator of a $\left(C_{0}\right)$-semigroup $\{T(t)\}$ satisfying $\|T(t)\| \leq M e^{\omega t}$ and $F: X \rightarrow X$ is a nonlinear operator which is continuously Fréchet differentiable on $X$ in the sense of $\S 1$. We show here that the linearized stability for semilinear case presented in [17, Proposition 4.17] can be obtained from our Theorem 2.1.

Corollary 2.2. Let $\bar{x} \in \mathscr{D}(L)$ be a stationary solution of $(S L)$, i.e., $(L+F) \bar{x}=$ 0 . Under the above setting, if the linearized operator $L+D F(\bar{x})$, which is the infinitesimal generator of a $\left(C_{0}\right)$-semigroup by the well-known perturbation theory, satisfies $\omega_{0}(L+D F(\bar{x}))<0$, then $\bar{x}$ is exponentially asymptotically stable in the sense that there exist $\eta>0, C \geq 1$, and $\alpha>0$ such that if $|x-\bar{x}|<\eta$, then a unique mild solution $u(t)$ of $(\mathrm{SL})$ :

$$
u(t)=T(t) x+\int_{0}^{t} T(t-s) F u(s) d s,
$$

exists for all $t \geq 0$ and $|u(t)-\bar{x}| \leq C e^{-\alpha t}|x-\bar{x}|$ for all $t \geq 0$.

\section{LINEARIZATION OF APPROXIMATING EQUATION}

In what follows, we write $J_{\lambda}$ instead of $J_{\lambda}^{A}$ for simplicity. Let $\left\{S_{\lambda}(t)\right\}$ be a semigroup on $X$ generated by $-A_{\lambda}$. It satisfies

$$
\left|S_{\lambda}(t) x-S_{\lambda}(t) y\right| \leq e^{\mu t}|x-y|, \quad \mu=\omega /(1-\lambda \omega) .
$$


It is well known that $u_{\lambda}(t):=S_{\lambda}(t) x, x \in X$, gives a unique classical solution $u_{\lambda} \in C^{1}([0, T] ; X)$ to the Cauchy problem

$$
\left\{\begin{array}{l}
(d / d t) u_{\lambda}(t)+A_{\lambda} u_{\lambda}(t)=0, \quad 0 \leq t \leq T, \\
u_{\lambda}(0)=x
\end{array}\right.
$$

for all $T>0$. Moreover, $u_{\lambda}$ satisfies

$$
u_{\lambda}(t)=e^{-\frac{1}{\lambda}} x+\frac{1}{\lambda} \int_{0}^{t} e^{\frac{\tau-t}{\lambda}} J_{\lambda} u_{\lambda}(\tau) d \tau .
$$

Recall further that $\lim _{\lambda \downarrow 0} S_{\lambda}(\cdot) u_{0}=S(\cdot) u_{0}$ in $C([0, T] ; X)$ for $u_{0} \in \overline{\mathscr{D}(A)}$, where $\{S(t)\}$ is a semigroup generated by $-A$. See $[2,3]$.

In this section we always assume the hypotheses (H1) and (H2). For $u \in$ $B_{r / 2}(\bar{u})$ and $0<\lambda<1 / 2 \omega$, one easily checks that $J_{\lambda} u \in B_{r}(\bar{u}) \cap \mathscr{D}(A)$. Thus there exists a linear operator $\partial A\left(J_{\lambda} u\right)$ such that $\partial A\left(J_{\lambda} u\right)+\omega I$ is $m$ accretive. By Lemma 1.2, one has $\partial A_{\lambda}(u) h=\left(\partial A\left(J_{\lambda} u\right)\right)_{\lambda} h$. Since $\left(\partial A\left(J_{\lambda} u\right)\right)_{\lambda}$ is Lipshitzian, it follows from Lemma 1.1 that the Yosida approximation $A_{\lambda}$ is Gâteaux differentiable at $u \in B_{r / 2}(\bar{u})$ and, denoting its Gâteaux derivative by $d A_{\lambda}(u)$, we have $d A_{\lambda}(u)=\partial A_{\lambda}(u)=\left(\partial A\left(J_{\lambda} u\right)\right)_{\lambda}$.

In what follows, we fix any $T>0$ and choose $\bar{r}$ such that $0<\bar{r}<$ $(r / 2) e^{-2 \omega T}$. Let $\lambda>0$ be sufficiently small so that $0<\lambda<\min \left\{1 / 2 \omega, \lambda_{\bar{u}}\right\}$ and let $x \in B_{F}(\bar{u})$. For the sake of simplicity of notation, we put $\partial A_{\lambda}(t) x:=$ $\partial A\left(J_{\lambda} S_{\lambda}(t) x\right)$. Since $\left|S_{\lambda}(t) x-\bar{u}\right|<r / 2$ for $0 \leq t \leq T$, we have

$$
d A_{\lambda}\left(S_{\lambda}(t) x\right)=\left(\partial A\left(J_{\lambda} S_{\lambda}(t) x\right)\right)_{\lambda}=\frac{1}{\lambda}\left(I-J_{\lambda}^{\partial A_{\lambda}(t) x}\right), \quad 0 \leq t \leq T .
$$

Now, we would like to consider the linearized equation for $\left(E_{\lambda}\right)$ :

$$
\left\{\begin{array}{l}
(d / d t) v_{\lambda}(t)+d A_{\lambda}\left(S_{\lambda}(t) x\right) v_{\lambda}(t)=0, \quad s \leq t \leq T \\
v_{\lambda}(s)=w
\end{array}\right.
$$

where $0 \leq s<T, w \in X$. Since $\partial A_{\lambda}(t) x+\omega I$ is $m$-accretive (by (H1)),

$$
\left|J_{\lambda}^{\partial A_{\lambda}(t) x} v-J_{\lambda}^{\partial A_{\lambda}(t) x} w\right| \leq \frac{1}{1-\lambda \omega}|v-w|, \quad \forall v, w \in X .
$$

Also, for $s<t<T, J_{\lambda} S_{\lambda}(t) x \in B_{r}(\bar{u}) \cap \mathscr{D}(A)$ and hence by (H2),

$$
\left|J_{\lambda}^{\partial A_{\lambda}(t) x} v-J_{\lambda}^{\partial A_{\lambda}(\tau) x} v\right| \leq \frac{\lambda}{1-\lambda \omega}\left|S_{\lambda}(t) x-S_{\lambda}(\tau) x\right| L_{\bar{u}}(|v|) .
$$

This shows that $t \mapsto J_{\lambda}^{\partial A_{\lambda}(t) x} v$ is continuous on [s,T]. Therefore by [3, Corollary 1.1, p.11], we have

Proposition 3.1. Let $x \in B_{r}(\bar{u})$. Then there exists a unique classical solution $v_{\lambda}(\cdot)=v_{\lambda}(\cdot ; s, w) \in C^{1}([s, T] ; X)$ to the Cauchy problem $\left(L_{\lambda} ; s, w\right)$. In addition, $v_{\lambda}$ satisfies

$$
v_{\lambda}(t)=e^{-\frac{t-s}{\lambda}} w+\frac{1}{\lambda} \int_{s}^{t} e^{\frac{\tau-t}{\lambda}} J_{\lambda}^{\partial A_{\lambda}(\tau) x} v_{\lambda}(\tau) d \tau
$$

and $\left|v_{\lambda}(t)\right| \leq e^{\mu(t-s)}|w|$ with $\mu=\omega /(1-\lambda \omega)$.

Let us define $\mathscr{S}_{\lambda}: X \rightarrow C([0, T] ; X)$ by

$$
\left(\mathscr{S}_{\lambda} x\right)(t):=S_{\lambda}(t) x \text { for } t \in[0, T] .
$$

We have the following representation of the solution of $\left(L_{\lambda} ; 0, w\right)$ : 
Proposition 3.2. $\mathscr{S}_{\lambda}$ is Gâteaux differentiable at each $x \in B_{r}(\bar{u})$ and the Gâteaux derivative $d \mathscr{S}_{\lambda}(x)$ represents a unique solution of $\left(L_{\lambda} ; 0, w\right)$ :

$$
d \mathscr{S}_{\lambda}(x) w=v_{\lambda}(\cdot ; 0, w) \equiv \text { the solution of }\left(L_{\lambda} ; 0, w\right) \text {. }
$$

Proof. At first, observe that $J_{\lambda}$ is Gâteaux differentiable on $B_{r / 2}(\bar{u})$ and $d J_{\lambda}(u)$ $=J_{\lambda}^{\partial A\left(J_{\lambda} u\right)}$ for $u \in B_{r / 2}(\bar{u})$. In view of (3.1) and (3.2), for sufficiently small $\alpha>0$,

$$
\begin{aligned}
& \left|\alpha^{-1}\left[S_{\lambda}(t)(x+\alpha w)-S_{\lambda}(t) x\right]-v_{\lambda}(t)\right| \\
& \leq \frac{1}{\lambda} \int_{0}^{t} e^{\frac{\tau-t}{\lambda}}\left|\alpha^{-1}\left[J_{\lambda} S_{\lambda}(\tau)(x+\alpha w)-J_{\lambda} S_{\lambda}(\tau) x\right]-d J_{\lambda}\left(S_{\lambda}(\tau) x\right) v_{\lambda}(\tau)\right| d \tau \\
& \leq \frac{1}{\lambda} \int_{0}^{t} e^{\frac{\tau-t}{\lambda}}\left|\alpha^{-1}\left[J_{\lambda} S_{\lambda}(\tau)(x+\alpha w)-J_{\lambda}\left(S_{\lambda}(\tau) x+\alpha v_{\lambda}(\tau)\right)\right]\right| d \tau \\
& \quad+\frac{1}{\lambda} \int_{0}^{t} e^{\frac{\tau-t}{\lambda}}\left|\alpha^{-1}\left[J_{\lambda}\left(S_{\lambda}(\tau) x+\alpha v_{\lambda}(\tau)\right)-J_{\lambda} S_{\lambda}(\tau) x\right]-d J_{\lambda}\left(S_{\lambda}(\tau) x\right) v_{\lambda}(\tau)\right| d \tau \\
& \leq \frac{1}{\lambda} \int_{0}^{t} e^{\frac{\tau-t}{\lambda}}(1-\lambda \omega)^{-1}\left|\alpha^{-1}\left[S_{\lambda}(\tau)(x+\alpha w)-S_{\lambda}(\tau) x\right]-v_{\lambda}(\tau)\right| d \tau \\
& \quad+\frac{1}{\lambda} \int_{0}^{t} e^{\frac{\tau-t}{\lambda}} h_{\alpha}(\tau) d \tau
\end{aligned}
$$

where $h_{\alpha}(\tau):=\left|\alpha^{-1}\left[J_{\lambda}\left(S_{\lambda}(\tau) x+\alpha v_{\lambda}(\tau)\right)-J_{\lambda} S_{\lambda}(\tau) x\right]-d J_{\lambda}\left(S_{\lambda}(\tau) x\right) v_{\lambda}(\tau)\right|$. Putting $\varphi(t):=e^{\frac{t}{\lambda}}\left|\alpha^{-1}\left[S_{\lambda}(t)(x+\alpha w)-S_{\lambda}(t) x\right]-v_{\lambda}(t)\right|$, we have

$$
\varphi(t) \leq \frac{1}{\lambda} \int_{0}^{t} e^{\frac{\tau}{\lambda}} h_{\alpha}(\tau) d \tau+\frac{1}{\lambda(1-\lambda \omega)} \int_{0}^{t} \varphi(\tau) d \tau .
$$

Then Gronwall's inequality yields that $\varphi(t) \leq \frac{1}{\lambda} \int_{0}^{t} e^{(t-\lambda \omega \xi) / \lambda(1-\lambda \omega)} h_{\alpha}(\xi) d \xi$. This leads to

$$
\sup _{0 \leq t \leq T}\left|\alpha^{-1}\left[S_{\lambda}(t)(x+\alpha w)-S_{\lambda}(t) x\right]-v_{\lambda}(t)\right| \leq \frac{1}{\lambda} e^{\mu T} \int_{0}^{T} h_{\alpha}(\tau) d \tau .
$$

Noting that $h_{\alpha}(\tau) \rightarrow 0$ as $\alpha \downarrow 0$ for $\tau \in[0, T]$ and

$$
\begin{aligned}
\left|h_{\alpha}(\tau)\right| & \leq\left|\alpha^{-1}\left[J_{\lambda}\left(S_{\lambda}(\tau)+\alpha v_{\lambda}(\tau)\right)-J_{\lambda} S_{\lambda}(\tau) x\right]\right|+\left|d J_{\lambda}\left(S_{\lambda}(\tau) x\right) v_{\lambda}(\tau)\right| \\
& \leq(1-\lambda \omega)^{-1}\left|v_{\lambda}(\tau)\right|+\left|J_{\lambda}^{\partial A_{\lambda}(\tau) x} v_{\lambda}(\tau)\right| \\
& \leq 2(1-\lambda \omega)^{-1}\left|v_{\lambda}(\tau)\right|
\end{aligned}
$$

one has, by Lebesgue's convergence theorem, $\int_{0}^{T} h_{\alpha}(\tau) d \tau \rightarrow 0$ as $\alpha \downarrow 0$. Thus the assertion follows.

Lemma 3.3. Fix $w \in X$. The mapping $z \mapsto d \mathscr{S}_{\lambda}(z) w$ is continuous from $B_{r}(\bar{u})$ into $C([0, T] ; X)$.

Proof. Let $z_{n} \rightarrow z_{0}$ in $B_{r}(\bar{u})$. We will show that $d \mathscr{S}_{\lambda}\left(z_{n}\right) w$ converges to $d \mathscr{S}_{\lambda}\left(z_{0}\right) w$ in $C([0, T] ; X)$. To do this, we employ Kisyński's technique. Denote by $c(X)$ the space of all convergent sequences $\mathbf{x}=\left\{x_{n}\right\}_{0}^{\infty}$ such that $x_{n} \rightarrow x_{0}$ in $X$. Thus $\mathbf{z}=\left\{z_{n}\right\}_{0}^{\infty} \in c(X) . c(X)$ is a Banach space under the 
norm $\|\mathbf{x}\|_{c(X)}=\sup _{n}\left|x_{n}\right|$. For $\mathbf{x}=\left\{x_{n}\right\} \in c(X)$, it follows from (H2) that

$$
\begin{aligned}
& \left|J_{\lambda}^{\partial A_{\lambda}(t) z_{n}} x_{n}-J_{\lambda}^{\partial A_{\lambda}(t) z_{0}} x_{0}\right| \\
& \quad \leq\left|J_{\lambda}^{\partial A_{\lambda}(t) z_{n}} x_{n}-J_{\lambda}^{\partial A_{\lambda}(t) z_{n}} x_{0}\right|+\left|J_{\lambda}^{\partial A_{\lambda}(t) z_{n}} x_{0}-J_{\lambda}^{\partial A_{\lambda}(t) z_{0}} x_{0}\right| \\
& \quad \leq(1-\lambda \omega)^{-1}\left(\left|x_{n}-x_{0}\right|+\lambda e^{\mu t}\left|z_{n}-z_{0}\right| L_{a}\left(\left|x_{0}\right|\right)\right), \quad \mu=\omega /(1-\lambda \omega) .
\end{aligned}
$$

From this, it is apparent that

$$
J_{\lambda}^{\partial A_{\lambda}(t) z_{n}} x_{n} \rightarrow J_{\lambda}^{\partial A_{\lambda}(t) z_{0}} x_{0} \quad \text { as } n \rightarrow \infty \text { uniformly in } t \in[0, T]
$$

Thus we can define $\mathbf{J}_{\lambda}^{\mathbf{z}}(t): c(X) \rightarrow c(X)$ by

$$
\mathbf{J}_{\lambda}^{\mathbf{z}}(t) \mathbf{x}=\left\{J_{\lambda}^{\partial A_{\lambda}(t) z_{n}} x_{n}\right\} \quad \text { for } \mathbf{x}=\left\{x_{n}\right\} \in c(X)
$$

It is easily seen that $\left\|\mathbf{J}_{\lambda}^{\mathbf{z}}(t) \mathbf{x}-\mathbf{J}_{\lambda}^{\mathbf{z}}(t) \mathbf{y}\right\|_{c(X)} \leq(1-\lambda \omega)^{-1}\|\mathbf{x}-\mathbf{y}\|_{c(X)}$. By (3.3) and [15, Lemma 5.1(i)], $t \mapsto \mathbf{J}_{\lambda}^{\mathbf{Z}}(t) \mathbf{x}$ is continuous. Thus by [3, Corollary 1.1, p.11], we conclude that for any $\mathbf{v}_{0} \in c(X)$, there exists a unique classical solution $\mathbf{v}_{\lambda}(\cdot)=\left\{v_{\lambda}^{n}(\cdot)\right\} \in C^{1}([0, T] ; c(X))$ to $(d / d t) \mathbf{v}_{\lambda}(t)+\lambda^{-1}\left(\mathbf{v}_{\lambda}(t)-\mathbf{J}_{\lambda}^{\mathbf{z}}(t) \mathbf{v}_{\lambda}(t)\right)=0$ and $\mathbf{v}_{\lambda}(0)=\mathbf{v}_{0}$. This implies that $\lim _{n \rightarrow \infty} v_{\lambda}^{n}(t)=v_{\lambda}^{0}(t)$ uniformly in $t \in[0, T]$ by [15, Lemma 5.1 (i)]. Taking the initial condition as $\mathbf{v}_{0}=\{w\}$, we conclude that $d \mathscr{S}_{\lambda}\left(z_{n}\right) w$ converges to $d \mathscr{S}_{\lambda}\left(z_{0}\right) w$ in $C([0, T] ; X)$ since $d \mathscr{S}_{\lambda}\left(z_{n}\right) w=v_{\lambda}^{n}$ by Proposition 3.2.

Lemma 3.4. For $x, y \in B_{\bar{r}}(\bar{u})$,

$$
\mathscr{S}_{\lambda} y-\mathscr{S}_{\lambda} x=\int_{0}^{1} d \mathscr{S}_{\lambda}(\theta y+(1-\theta) x)(y-x) d \theta \quad \text { in } C([0, T] ; X) \text {. }
$$

Proof. Since $\theta y+(1-\theta) x \in B_{\bar{r}}(\bar{u})$ for $\theta \in[0,1]$, by Proposition 3.2, we have

$$
\begin{aligned}
\left(\frac{d}{d \theta}\right)^{+} & \mathscr{S}_{\lambda}(\theta y+(1-\theta) x) \\
= & \lim _{h \downarrow 0} h^{-1}\left[\mathscr{S}_{\lambda}(x+\theta(y-x)+h(y-x))-\mathscr{S}_{\lambda}(x+\theta(y-x))\right] \\
& =d \mathscr{S}_{\lambda}(x+\theta(y-x))(y-x) .
\end{aligned}
$$

By Lemma 3.3, $\theta \mapsto(d / d \theta)+\mathscr{S}_{\lambda}(\theta y+(1-\theta) x)$ is continuous from $[0,1]$ to $C([0, T] ; X)$. Integrating the above over $[0,1]$ yields the result.

Lemma 3.5. Let $x \in B_{r}(\bar{u})$ and $0 \leq t_{0}<T$. Let $v_{\lambda}^{x}(t)$ and $v_{\lambda}^{\bar{u}}(t)$ be solutions to $\left(L_{\lambda} ; t_{0}, w\right)$ with operators $d A_{\lambda}\left(S_{\lambda}(t) x\right)$ and $d A_{\lambda}(\bar{u})$, respectively. Then

$$
\left|v_{\lambda}^{x}(t)-v_{\lambda}^{\bar{u}}(t)\right| \leq(1-\lambda \omega)^{-1} e^{\mu t}\left(t-t_{0}\right)|x-\bar{u}| L_{\bar{u}}\left(e^{\mu T}|w|\right) \quad \text { for } t_{0} \leq t \leq T
$$


Proof. By (3.2) and (H2), we have

$$
\begin{aligned}
\left|v_{\lambda}^{x}(t)-v_{\lambda}^{\bar{u}}(t)\right| \leq & \frac{1}{\lambda} \int_{t_{0}}^{t} e^{\frac{\tau-t}{\lambda}}\left|J_{\lambda}^{\partial A_{\lambda}(\tau) x} v_{\lambda}^{x}(\tau)-J_{\lambda}^{\partial A_{\lambda}(\tau) \bar{u}} v_{\lambda}^{\bar{u}}(\tau)\right| d \tau \\
\leq & \frac{1}{\lambda} \int_{t_{0}}^{t} e^{\frac{\tau-t}{\lambda}}\left|J_{\lambda}^{\partial A_{\lambda}(\tau) x} v_{\lambda}^{x}(\tau)-J_{\lambda}^{\partial A_{\lambda}(\tau) \bar{u}}(\tau) v_{\lambda}^{x}(\tau)\right| d \tau \\
& +\frac{1}{\lambda} \int_{t_{0}}^{t} e^{\frac{\tau-t}{\lambda}}\left|J_{\lambda}^{\partial A_{\lambda}(\tau) \bar{u}} v_{\lambda}^{x}(\tau)-J_{\lambda}^{\partial A_{\lambda}(\tau) \bar{u}} v_{\lambda}^{\bar{u}}(\tau)\right| d \tau \\
\leq & \int_{t_{0}}^{t} e^{\frac{\tau-t}{\lambda}}(1-\lambda \omega)^{-1} e^{\mu \tau}|x-\bar{u}| L_{\bar{u}}\left(e^{\mu\left(t-t_{0}\right)}|w|\right) d \tau \\
& +\frac{1}{\lambda} \int_{t_{0}}^{t} e^{\frac{\tau-t}{\lambda}}(1-\lambda \omega)^{-1}\left|v_{\lambda}^{x}(\tau)-v_{\lambda}^{\bar{u}}(\tau)\right| d \tau .
\end{aligned}
$$

Putting $\varphi(t)=e^{\frac{1}{\lambda}}\left|v_{\lambda}^{x}(t)-v_{\lambda}^{\bar{u}}(t)\right|$ yields

$$
\varphi(t) \leq \lambda|x-\bar{u}| L_{\bar{u}}\left(e^{\mu T}|w|\right)\left(e^{t / \lambda(1-\lambda \omega)}-e^{t_{0} / \lambda(1-\lambda \omega)}\right)+\frac{1}{\lambda(1-\lambda \omega)} \int_{t_{0}}^{t} \varphi(\tau) d \tau .
$$

Then we deduce from Gronwall's inequality that

$$
\varphi(t) \leq|x-\bar{u}| L_{\bar{u}}\left(e^{\mu T}|w|\right)(1-\lambda \omega)^{-1} e^{t / \lambda(1-\lambda \omega)}\left(t-t_{0}\right),
$$

and the desired inequality readily holds.

\section{Proofs of Theorem 2.1 AND Corollary 2.2}

Proof of Theorem 2.1. Let $\{T(t)\}$ be a $\left(C_{0}\right)$-semigroup generated by $-\partial A(\bar{u})$. Since $\omega_{0}(\partial A(\bar{u}))<0$, there exist $\gamma>0$ and $M>0$ such that $\|T(t)\| \leq M e^{-\gamma t}$. Put $U(t)=e^{\gamma t} T(t)$. Then $\|x\|:=\sup _{t \geq 0}|U(t) x|$ defines a norm equivalent to the original norm. In fact, $|\cdot| \leq\|\cdot\| \leq M|\cdot|$. It readily follows that $\|T(t) x\| \leq e^{-\gamma t}\|x\|$ and hence, as is well known, $\partial A(\bar{u})-\gamma I$ is $m$-accretive in the renormed Banach space $(X,\|\cdot\|)$. That implies that $d A_{\lambda}(\bar{u})-\gamma_{\lambda} I$ is $m$-accretive in $(X,\|\cdot\|)$, where $\gamma_{\lambda}=\gamma /(1+\lambda \gamma)$. Fix $T>0$. Denoting by $v_{\lambda}^{a}(\cdot)$ the solution of $\left(L_{\lambda} ; 0, w\right)$ with operator $d A_{\lambda}(\bar{u})$ in place of $d A_{\lambda}\left(S_{\lambda}(t) x\right)$, one has

$$
\left\|v_{\lambda}^{\bar{u}}(t)\right\| \leq e^{-\gamma_{\lambda} t}\|w\|, \quad w \in X, \quad t \geq 0 .
$$

Let $\varepsilon_{0} \in(0, \gamma)$. There exists a $\lambda_{0}>0$ such that $0<\lambda<\lambda_{0}$ implies $0<\gamma-\gamma_{\lambda}<$ $\varepsilon_{0}$. In the sequel, $\lambda>0$ is taken so small that $0<\lambda<\min \left\{\lambda_{0}, \lambda_{\bar{u}}, 1 / 2 \omega\right\}$. Take $\alpha \in\left(0, \gamma-\varepsilon_{0}\right)$ and choose $\varepsilon \in\left(0, e^{-\alpha T}-e^{-\left(\gamma-\varepsilon_{0}\right) T}\right)$. Let $\bar{r}$ be as in $\S 3$, i.e., $0<\bar{r}<(r / 2) e^{-2 \omega T}$. And then take $\bar{\eta}>0$ such that $0<\bar{\eta}<$ $\min \left\{\bar{r}, \varepsilon / 2 M e^{2 \omega T} T L_{\bar{u}}\left(e^{2 \omega T}\right)\right\}$.

If $\|x-\bar{u}\|<\bar{\eta}$ (which implies $|x-\bar{u}|<\bar{\eta}$ ), then, by Lemma 3.5 and (4.1), the following estimate holds:

$$
\begin{aligned}
\left\|\left[d \mathscr{S}_{\lambda}(x) w\right](T)\right\| & \leq\left\|v_{\lambda}^{x}(T ; 0, w)-v_{\lambda}^{\bar{a}}(T ; 0, w)\right\|+\left\|v_{\lambda}^{\bar{u}}(T ; 0, w)\right\| \\
& \leq M\left|v_{\lambda}^{x}(T ; 0, w)-v_{\lambda}^{a}(T ; 0, w)\right|+\left\|v_{\lambda}^{\bar{u}}(T ; 0, w)\right\| \\
& \leq M(1-\lambda \omega)^{-1} e^{\mu T} T|x-\bar{u}| L_{\bar{u}}\left(e^{\mu T}|w|\right)+e^{-\gamma_{\lambda} T}\|w\| \\
& \leq 2 M e^{2 \omega T} T \bar{\eta} L_{\bar{u}}\left(e^{2 \omega T}\|w\|\right)+e^{-\left(\gamma-\varepsilon_{0}\right) T}\|w\| .
\end{aligned}
$$


This implies that $\sup _{\|w\| \leq 1}\left\|\left[d \mathscr{S}_{\lambda}(x) w\right](T)\right\|<e^{-\alpha T}$ by way of choosing $\alpha$. Since $v \mapsto d \mathscr{S}_{\lambda}(x) v$ is linear, we obtain

$$
\left\|\left[d \mathscr{S}_{\lambda}(x) v\right](T)\right\| \leq e^{-\alpha T}\|v\|, \quad \forall v \in X \text { whenever }\|x-\bar{u}\|<\bar{\eta} .
$$

Let $\|x-\bar{u}\|<\bar{\eta}$ and $\theta \in[0,1]$. Since $\|\theta x+(1-\theta) \bar{u}-\bar{u}\|<\bar{\eta}$, by (4.2), we get

$$
\left\|\left[d \mathscr{S}_{\lambda}(\theta x+(1-\theta) \bar{u})(x-\bar{u})\right](T)\right\| \leq e^{-\alpha T}\|x-\bar{u}\| .
$$

Noting that $S_{\lambda}(t) \bar{u}=\bar{u}$, it follows from Lemma 3.4 that

$$
\left\|S_{\lambda}(T) x-\bar{u}\right\| \leq e^{-\alpha T}\|x-\bar{u}\| \quad \text { if }\|x-\bar{u}\|<\bar{\eta} .
$$

Now, let $\eta=\bar{\eta} / M$ and let $u_{0} \in \overline{\mathscr{D}(A)},\left|u_{0}-\bar{u}\right|<\eta$. Then $\left\|u_{0}-\bar{u}\right\|<\bar{\eta}$ and thus using (4.3) repeatedly, we have, for any integer $k$,

$$
\left\|S_{\lambda}(k T) u_{0}-\bar{u}\right\| \leq e^{-k \alpha T}\left\|u_{0}-\bar{u}\right\| .
$$

For any $t>T$, let $k:=[t / T]([\cdot]$ stands for the Gaussian bracket). Then we have

$$
\begin{aligned}
\left\|S_{\lambda}(t) u_{0}-\bar{u}\right\| & \leq M\left|S_{\lambda}(t-k T) S_{\lambda}(k T) u_{0}-\bar{u}\right| \\
& \left.\leq M e^{\mu T}\left|S_{\lambda}(k T) u_{0}-\bar{u}\right| \leq M e^{\mu T} e^{-k \alpha T}\left\|u_{0}-\bar{u}\right\| \quad \text { by }(4.4)\right) \\
& \leq M e^{\mu T} e^{-\alpha(t-T)}\left\|u_{0}-\bar{u}\right\| \leq M e^{(2 \omega+\alpha) T} e^{-\alpha t}\left\|u_{0}-\bar{u}\right\| .
\end{aligned}
$$

It readily follows that $\left|S_{\lambda}(t) u_{0}-\bar{u}\right| \leq C e^{-\alpha t}\left|u_{0}-\bar{u}\right|$ with $C=M^{2} e^{(\bar{z} \omega+\alpha) T}$. Hence letting $\lambda \downarrow 0$, we obtain $\left|S(t) u_{0}-\bar{u}\right| \leq C e^{-\alpha t}\left|u_{0}-\bar{u}\right|$ for all $t>T$. For $0 \leq t \leq T$, it is easy to see that $\left|S(t) u_{0}-\bar{u}\right| \leq e^{(\alpha+\omega) T} e^{-\alpha t}\left|u_{0}-\bar{u}\right|$. Thus the proof is complete.

Proof of Corollary 2.2. Let $\bar{x} \in \mathscr{D}(L)$ satisfy $(L+F) \bar{x}=0$. Fix $r_{0}>0$ such that $|\bar{x}|<r_{0}$ and define

$$
F_{0}(\phi):= \begin{cases}F(\phi) & \text { if }|\phi| \leq r_{0} \\ F\left(r_{0} \phi /|\phi|\right) & \text { if }|\phi|>r_{0} .\end{cases}
$$

Then as mentioned in Remark 1.1, $F_{0}: X \rightarrow X$ is continuously Fréchet differentiable on $B_{r_{0}}(0)$, and globally Lipschitz continuous. We denote its Lipschitz constant by $\left\|F_{0}\right\|_{\text {Lip }}$.

Let us define an operator $A$ by

$$
A u=-\left(L+F_{0}\right) u \text { for } u \in \mathscr{D}(A)=\mathscr{D}(L) .
$$

By the standard renorming $|u|:=\sup _{t>0}\left\|e^{-\omega t} T(t) u\right\|$ (using the same notation as the original norm) and then by regarding $-\left(L+F_{0}\right)$ as $-(L-\omega I)-\left(F_{0}+\omega I\right)$, we may assume without loss of generality that $\{T(t)\}$ is a contraction semigroup and so $-L$ is $m$-accretive. (See [10, Proposition 2.5].)

In the following, we will assume $-L$ is $m$-accretive and check all the hypotheses assumed in Theorem 2.1.

Proposition 4.1. $A+\left\|F_{0}\right\|_{\text {Lip }} I$ is a densely defined m-accretive operator in $X$. Proof. The following calculation shows that $A+\left\|F_{0}\right\|_{\text {Lip } I} I$ is accretive:

$$
\begin{aligned}
|u-v+\lambda(A u-A v)| & \geq|u-v-\lambda L(u-v)|-\lambda\left|F_{0}(u)-F_{0}(v)\right| \\
& \geq|u-v|-\lambda\left\|F_{0}\right\|_{\text {Lip }}|u-v| .
\end{aligned}
$$


To show the range condition $\mathscr{R}(I+\lambda A)=X$ for sufficiently small $\lambda>$ 0 , take any $x \in X$. For $z \in X$, define $T: X \rightarrow \mathscr{D}(L) \subset X$ by $T z=$ $(I-\lambda L)^{-1}\left(x+\lambda F_{0}(z)\right)$. Then obviously, $|T z-T \hat{z}| \leq \lambda\left\|F_{0}\right\|_{\text {Lip }}|z-\hat{z}|$, and so $T$ is a strict contraction for small $\lambda>0$. Hence there exists a unique $u \in \mathscr{D}(L)$ such that $u-\lambda L u=x+\lambda F_{0}(u)$, which shows the range condition. It is known that $\mathscr{D}(A)=\mathscr{D}(L)$ is densely defined since $L$ is the infinitesimal generator of a $\left(C_{0}\right)$-contraction semigroup.

Now, take $r>0$ such that $|\bar{x}|+r<r_{0}$. Then $x \in B_{r}(\bar{x})$ implies $x \in$ $B_{r_{0}}(0)$. Taking this into account, we can define a linear operator $\partial A(u)$ for $u \in \mathscr{D}(A) \cap B_{r}(\bar{x})$ by

$$
\partial A(u) v=-L v-D F_{0}(u) v \quad \text { for } v \in \mathscr{D}(\partial A(u))=\mathscr{D}(L)
$$

By the same reason as the above proposition, we have

Proposition 4.2. $\partial A(u)+\left\|D F_{0}(u)\right\| I$ is m-accretive in $X$ for $u \in \mathscr{D}(A) \cap B_{r}(\bar{x})$.

The next proposition justifies the notation $\partial A(u)$, which is used to express the proto-derivative.

Proposition 4.3. $\mathscr{G}(\partial A(u))=\lim _{t \downarrow 0} t^{-1}[\mathscr{G}(A)-(u, A u)]$ for $u \in \mathscr{D}(A) \cap B_{r}(\bar{x})$. Proof. Firstly, we show that

$$
\mathscr{G}(\partial A(u)) \subset \liminf _{t \downarrow 0} t^{-1}[\mathscr{G}(A)-(u, A u)] .
$$

Let $(h, k) \in \mathscr{G}(\partial A(u))$. Then $k=-L h-D F_{0}(u) h$. Take any sequence $t_{n} \downarrow 0$ and let $h_{n}:=h$ and $k_{n}:=-L h-t_{n}^{-1}\left[F_{0}\left(u+t_{n} h\right)-F_{0}(u)\right]$. Then $k_{n} \rightarrow$ $-L h-D F_{0}(u) h=k$. This shows that $(h, k) \in \liminf _{t \downarrow 0} t^{-1}[\mathscr{G}(A)-(u, A u)]$.

Next we show that

$$
\limsup _{t \downarrow 0} t^{-1}[\mathscr{G}(A)-(u, A u)] \subset \mathscr{G}(\partial A(u)) .
$$

Let $(\hat{h}, k) \in \lim \sup _{t \downarrow 0} t^{-1}[\mathscr{G}(A)-(u, A u)]$. Then there exist a null sequence $t_{n} \downarrow 0$ and $\left(h_{n}, k_{n}\right) \rightarrow(h, k)$ such that $\left(u+t_{n} h_{n}, A u+t_{n} k_{n}\right) \in \mathscr{G}(A)$. Since $F_{0}$ is Lipschitzian,

$$
\begin{aligned}
\mid t_{n}^{-1}[ & \left.F_{0}\left(u+t_{n} h_{n}\right)-F_{0}(u)\right]-D F_{0}(u) h \mid \\
& \leq\left|t_{n}^{-1}\left[F_{0}\left(u+t_{n} h_{n}\right)-F_{0}\left(u+t_{n} h\right)\right]\right|+\left|t_{n}^{-1}\left[F_{0}\left(u+t_{n} h\right)-F_{0}(u)\right]-D F_{0}(u) h\right| \\
& \leq\left\|F_{0}\right\|_{L i p}\left|h_{n}-h\right|+\left|t_{n}^{-1}\left[F_{0}\left(u+t_{n} h\right)-F_{0}(u)\right]-D F_{0}(u) h\right| \rightarrow 0 \text { as } n \rightarrow \infty .
\end{aligned}
$$

Noting that $h_{n} \in \mathscr{D}(L)$ (by the linearity of $\mathscr{D}(L)$ ), the above calculation shows that $h_{n} \in \mathscr{D}(L) \rightarrow h$ and $L h_{n}=-k_{n}+t_{n}^{-1}\left[F_{0}\left(u+t_{n} h_{n}\right)-F_{0}(u)\right] \rightarrow$ $-k+D F_{0}(u) h$. Since $L$ is closed, we conclude that $h \in \mathscr{D}(L)$ and $k=$ $-L h+D F_{0}(u) h$, i.e., $(h, k) \in \mathscr{G}(\partial A(u))$.

Corollary 4.4. $\partial A(u)+\left\|F_{0}\right\|_{\text {Lip }} I$ is $m$-accretive in $X$.

Proof. Let us set $B=A+\left\|F_{0}\right\|_{\text {Lip }} I$ and $\partial B(u)=\partial A(u)+\left\|F_{0}\right\|_{\text {Lip }} I$. Observing that $(h, k) \in \mathscr{G}(\partial B(u))$ iff $\left(h, k-\left\|F_{0}\right\|_{\text {Lip }} h\right) \in \mathscr{G}(\partial A(u))$ and that $(h, k) \in$ $\lim _{t \downarrow 0} t^{-1}[\mathscr{G}(B)-(u, B u)]$ iff $\left(h, k-\left\|F_{0}\right\|_{\text {Lip }} h\right) \in \lim _{t \downarrow 0} t^{-1}[\mathscr{G}(A)-(u, A u)]$, it follows from Proposition 4.3 that

$$
\mathscr{G}(\partial B(u))=\lim _{t \downarrow 0} t^{-1}[\mathscr{G}(B)-(u, B u)] .
$$


Since the right-hand side is accretive, so is the left. From the fact that $\partial A(u)+$ $\left\|D F_{0}(u)\right\| I$ is $m$-accretive, it is not difficult to see that for any $f \in X$ and sufficiently small $\mu>0$, there exists an $h \in \mathscr{D}(\partial A(u))$ such that

$$
h+\mu\left(\partial A(u) h+\left\|F_{0}\right\|_{\text {Lip }} h\right)=f \text {. }
$$

Finally we have

Proposition 4.5. There exists a nondecreasing function $L_{\bar{x}}:[0, \infty) \rightarrow[0, \infty)$ such that

$$
\left|J_{\lambda}^{\partial A(z)} v-J_{\lambda}^{\partial A(u)} v\right| \leq \lambda|z-u| L_{\bar{x}}(|v|)
$$

for $\lambda>0, z, u \in B_{r}(\bar{x}) \cap \mathscr{D}(A)$, and $v \in X$.

Proof. It readily follows that

$$
\left|J_{\lambda}^{\partial A(z)} v-J_{\lambda}^{\partial A(u)} v\right|=\lambda\left|D F_{0}(z) v-D F_{0}(u) v\right| \leq \lambda d(r)|z-u||v|
$$

for $\lambda>0, z, u \in B_{r}(\bar{x}) \cap \mathscr{D}(A)$, and $v \in X$.

Now, we are back to the proof of Corollary 2.2. The above propositions guarantee that the hypotheses $(\mathrm{H} 1)$ and $(\mathrm{H} 2)$ are fulfilled. Noting that $-\partial A(\bar{x})=$ $L+D F_{0}(\bar{x})=L+D F(\bar{x})$, if $\omega_{0}(L+D F(\bar{x}))<0$, then we conclude from Theorem 2.1 that there exist $\eta>0, M \geq 1$ and $\alpha>0$ such that if $|x-\bar{x}|<\eta$, then $|S(t) x-\bar{x}| \leq M e^{-\alpha t}|x-\bar{x}|$, where $\{S(t)\}$ is a semigroup generated by $-A=$ $L+F_{0}$. It is known that $S(t)$ satisfies $S(t)=T(t) x+\int_{0}^{t} T(t-s) F_{0} S(s) x d s$. (See the proof of Proposition 3.18 of [19].) Take $\eta>0$ sufficiently small so that $M \eta<r$. Then $S(s) x \in B_{r}(\bar{x})$, which implies $S(s) x \in B_{r_{0}}(0)$. Therefore, $S(t)$ satisfies the variation of constants formula (2.2). The uniqueness of the mild solution is well-known. The proof of Corollary 2.2 is now completed.

\section{AGE-DEPENDENT POPULATION DYNAMICS}

In this section, we treat a problem of age-dependent population dynamics rather general form than usual. Let $(E,|\cdot|)$ be a Banach space and $L^{1}:=$ $L^{1}(0, \infty ; E)$ be a Bochner integrable function space, which norm is denoted by $\|\cdot\|_{L^{1}}$. Given two mappings $F: L^{1} \rightarrow E$ and $G: L^{1} \rightarrow L^{1}$, we consider the following partial differential equation:

$$
\left\{\begin{array}{l}
\frac{\partial}{\partial t} u(t, a)+\frac{\partial}{\partial a} u(t, a)=G(u(t, \cdot))(a), \quad t \geq 0, a \geq 0, \\
u(t, 0)=F(u(t, \cdot)), \quad t \geq 0 .
\end{array}\right.
$$

The usual model is obtained as follows: $E=\mathbb{R}^{n}$. For $i=1, \cdots, n$, define $K_{i}, J_{i}: L^{1} \rightarrow[0, \infty)$ by $K_{i} \phi=\int_{0}^{\infty} k_{i}(a) \phi(a) d a, J_{i} \phi=\int_{0}^{\infty} j_{i}(a) \phi(a) d a$, respectively, where $k_{i}, j_{i}:[0, \infty) \rightarrow \mathscr{L}\left(\mathbb{R}^{n},[0, \infty)\right)$ are given mappings. Then define $F: L^{1} \rightarrow \mathbb{R}^{n}, G: L^{1} \rightarrow L^{1}$ by taking their $i$-th component as follows:

$$
\begin{aligned}
F(\phi)_{i} & =\int_{0}^{\infty} \beta_{i}\left(a, K_{i} \phi\right) \phi_{i}(a) d a \text { for } \phi=\left(\phi_{i}\right) \in L^{1}, \\
G(\phi)_{i}(a) & =-\mu_{i}\left(a, J_{i} \phi\right) \phi_{i}(a) \quad \text { a.e. } a>0 \text { for } \phi=\left(\phi_{i}\right) \in L^{1},
\end{aligned}
$$

where $\beta_{i}, \mu_{i}:[0, \infty) \times[0, \infty) \rightarrow[0, \infty)$ are given functions. See [17] for details. 
In the following, we assume that $F: L^{1} \rightarrow E, G: L^{1} \rightarrow L^{1}$ are continuously Fréchet differentiable in the sense of $\S 1$, i.e.,

(F) For any $\phi \in L^{1}$, there exists a $D F(\phi) \in \mathscr{L}\left(L^{1}, E\right)$ such that

$$
F(\phi+h)=F(\phi)+D F(\phi) h+o_{F}(h), \quad h \in L^{1},
$$

where $o_{F}: L^{1} \rightarrow E,\left|o_{F}(h)\right| \leq b_{F}(r)\|h\|_{L^{1}}$ for $\|h\|_{L^{1}} \leq r$, and $b_{F}$ : $[0, \infty) \rightarrow[0, \infty)$ is a continuous increasing function satisfying $b_{F}(0)=$

0 ; and there exists a continuous increasing function $d_{F}:[0, \infty) \rightarrow$ $[0, \infty)$ such that

$$
\|D F(\phi)-D F(\psi)\|_{\mathscr{L}\left(L^{1}, E\right)} \leq d_{F}(r)\|\phi-\psi\|_{L^{1}} \text { for }\|\phi\|_{L^{1}},\|\psi\|_{L^{1}} \leq r .
$$

(G) For any $\phi \in L^{1}$, there exists a $D G(\phi) \in \mathscr{L}\left(L^{1}, L^{1}\right)$ such that

$$
G(\phi+h)=G(\phi)+D G(\phi) h+o_{G}(h), \quad h \in L^{1},
$$

where $o_{G}: L^{1} \rightarrow L^{1},\left\|o_{G}(h)\right\|_{L^{1}} \leq b_{G}(r)\|h\|_{L^{1}}$ for $\|h\|_{L^{1}} \leq r$, and $b_{G}$ : $[0, \infty) \rightarrow[0, \infty)$ is a continuous increasing function satisfying $b_{G}(0)=$ 0 ; and there exists a continuous increasing function $d_{G}:[0, \infty) \rightarrow$ $[0, \infty)$ such that

$$
\|D G(\phi)-D G(\psi)\|_{\mathscr{L}\left(L^{1}, L^{1}\right)} \leq d_{G}(r)\|\phi-\psi\|_{L^{1}} \text { for }\|\phi\|_{L^{1}},\|\psi\|_{L^{1}} \leq r .
$$

Let $\bar{u}$ be a stationary solution of $(\mathrm{P})$, i.e., $\bar{u} \in W^{1,1}:=W^{1,1}(0, \infty ; E)$, $\bar{u}(0)=F(\bar{u})$, and $\bar{u}^{\prime}=G(\bar{u})$, where ' stands for $d / d a$ when the variable of functions in $W^{1,1}$ is represented by $a$. Fix $r_{0}>0$ such that $\|\bar{u}\|_{L^{1}}<r_{0}$. Then define the radial truncations $F_{0}$ and $G_{0}$ by

$$
\begin{aligned}
F_{0}(\phi) & := \begin{cases}F(\phi) & \text { if }\|\phi\|_{L^{1}} \leq r_{0}, \\
F\left(r_{0} \phi /\|\phi\|_{L^{1}}\right) & \text { if }\|\phi\|_{L^{1}}>r_{0},\end{cases} \\
G_{0}(\phi) & := \begin{cases}G(\phi) & \text { if }\|\phi\|_{L^{1}} \leq r_{0}, \\
G\left(r_{0} \phi /\|\phi\|_{L^{1}}\right) & \text { if }\|\phi\|_{L^{1}}>r_{0} .\end{cases}
\end{aligned}
$$

As in Remark 1.1, $F_{0}$ and $G_{0}$ are globally Lipschitz continuous and continuously Fréchet differentiable on the ball $B_{r_{0}}(0)$ in $L^{1}$.

Now define an operator $A$ on $L^{1}$ by

$$
A \phi=\phi^{\prime}-G_{0}(\phi) \text { for } \phi \in \mathscr{D}(A):=\left\{\phi \in W^{1,1} \mid \phi(0)=F_{0}(\phi)\right\} .
$$

We will observe that the hypotheses $(\mathrm{H} 1)$ and $(\mathrm{H} 2)$ in $\S 2$ are satisfied. At first, we have

Proposition 5.1. With $\omega=\left\|F_{0}\right\|_{\text {Lip }}+\left\|G_{0}\right\|_{\text {Lip }}, A+\omega I$ is a densely defined $m$ accretive operator in $L^{1}$.

Proof. Firstly, we will show that $\mathscr{R}(I+\lambda A)=L^{1}, 0<\lambda<1 / \omega$. We must show that for any $\psi \in L^{1}$, there exists a $\phi \in W^{1,1}$ such that $\phi+\lambda \phi^{\prime}-\lambda G_{0}(\phi)=\psi$ and $\phi(0)=F_{0}(\phi)$, which is equivalent to the integral equation:

$$
\phi(x)=F_{0}(\phi) e^{-\frac{x}{\lambda}}+\int_{0}^{x} e^{-\frac{x-t}{\lambda}}\left[\frac{1}{\lambda} \psi(t)+G_{0}(\phi)(t)\right] d t .
$$

Thus for $\phi \in L^{1}$, we define $K: L^{1} \rightarrow L^{1}$ by

$$
(K \phi)(x)=F_{0}(\phi) e^{-\frac{x}{\lambda}}+\int_{0}^{x} e^{-\frac{x-t}{\lambda}}\left[\frac{1}{\lambda} \psi(t)+G_{0}(\phi)(t)\right] d t
$$


and seek the fixed point of $K$. It readily follows that

$$
\begin{aligned}
\| K \phi- & K \hat{\phi} \|_{L^{1}} \leq \int_{0}^{\infty}\left|F_{0}(\phi)-F_{0}(\hat{\phi})\right| e^{-\frac{x}{\lambda}} d x \\
& +\int_{0}^{\infty} \int_{0}^{x} e^{-\frac{x-t}{\lambda}}\left|G_{0}(\phi)(t)-G_{0}(\hat{\phi})(t)\right| d t d x \\
\leq & \lambda\left\|F_{0}\right\|_{L i p}\|\phi-\hat{\phi}\|_{L^{1}}+\lambda \int_{0}^{\infty}\left|G_{0}(\phi)(t)-G_{0}(\hat{\phi})(t)\right| d t \leq \lambda \omega\|\phi-\hat{\phi}\|_{L^{1}} .
\end{aligned}
$$

Hence for $0<\lambda \omega<1$, the fixed point of $K$ exists by the Banach contraction mapping theorem.

Next, we will show that $A+\omega I$ is accretive. Let $\psi=(I+\lambda A) \phi$ and $\hat{\psi}=(I+\lambda A) \hat{\phi}$. Then by $(5.1)$,

$$
\begin{aligned}
\|\phi-\hat{\phi}\|_{L^{1}} \leq & \int_{0}^{\infty} e^{-\frac{x}{\lambda}}\left|F_{0}(\phi)-F_{0}(\hat{\phi})\right| d x+\frac{1}{\lambda} \int_{0}^{\infty} \int_{0}^{x} e^{-\frac{x-t}{\lambda}}|\psi(t)-\hat{\psi}(t)| d t d x \\
& +\int_{0}^{\infty} \int_{0}^{x} e^{-\frac{x-t}{\lambda}}\left|G_{0}(\phi)(t)-G_{0}(\hat{\phi})(t)\right| d t d x \\
\leq & \lambda\left\|F_{0}\right\|_{L i p}\|\phi-\hat{\phi}\|_{L^{1}}+\lambda\left\|G_{0}\right\|_{\text {Lip }}\|\phi-\hat{\phi}\|_{L^{1}}+\|\psi-\hat{\psi}\|_{L^{1}} .
\end{aligned}
$$

Therefore, $(1-\lambda \omega)\|\phi-\hat{\phi}\|_{L^{1}} \leq\|\psi-\hat{\psi}\|_{L^{1}}$. Finally, it is shown that the domain of $A$ is dense similarly to Webb [17, Proposition 3.8].

Choose $r>0$ such that $\|\bar{u}\|_{L^{1}}+r<r_{0}$. Note that $u \in B_{r}(\bar{u})$ implies that $u \in B_{r_{0}}(0)$. For $u \in \mathscr{D}(A) \cap B_{r}(\bar{u})$, we define

$$
\partial A(u) h=h^{\prime}-D G(u) h \quad \text { for } h \in \mathscr{D}(\partial A(u)):=\left\{h \in W^{1,1} \mid h(0)=D F(u) h\right\} .
$$

Then by the same reason as the above proposition, we have the following

Proposition 5.2. With $\omega_{u}:=\|D F(u)\|_{\mathscr{L}\left(L^{1}, E\right)}+\|D G(u)\|_{\mathscr{L}\left(L^{1}, L^{1}\right)}, \partial A(u)+\omega_{u} I$ is $m$-accretive in $L^{1}$.

As in the previous section, we write $J_{\lambda}=J_{\lambda}^{A}$.

Lemma 5.3. Let $S:=\left\{v \in L^{1} \mid J_{\lambda} v \in B_{r}(\bar{u}), 0<\lambda<1 / 2 \omega\right\}$. Then $J_{\lambda}$ is Gâteaux differentiable on $S$ and $d J_{\lambda}(v) h=J_{\lambda}^{\partial A\left(J_{\lambda} v\right)} h$ for $v \in S, h \in L^{1}$, $0<\lambda<1 / 2 \omega$.

Remark 5.1. It is easily checked that $B_{r / 2}(\bar{u}) \subset S$.

Proof. Let $v \in S$. Recall that $\phi=J_{\lambda} v$ is given by (5.1). Set $\phi_{t}=J_{\lambda}(v+t h)$ and $\psi=J_{\lambda}^{\partial A\left(J_{\lambda} v\right)} h$. Then these satisfy

$$
\begin{aligned}
\phi_{t}(x) & =e^{-\frac{x}{\lambda}} F_{0}\left(\phi_{t}\right)+\int_{0}^{x} e^{-\frac{x-s}{\lambda}}\left[\frac{1}{\lambda}(v(s)+t h(s))+G_{0}\left(\phi_{t}\right)(s)\right] d s, \\
\psi(x) & =e^{-\frac{x}{\lambda}} D F(\phi)(\psi)+\int_{0}^{x} e^{-\frac{x-s}{\lambda}}\left[\frac{1}{\lambda} h(s)+D G(\phi)(\psi)(s)\right] d s
\end{aligned}
$$


Therefore, the following estimate holds:

$$
\begin{aligned}
& \left\|t^{-1}\left(\phi_{t}-\phi\right)-\psi\right\|_{L^{1}} \\
& \leq \int_{0}^{\infty} e^{-\frac{x}{\lambda}} d x\left|t^{-1}\left(F_{0}\left(\phi_{t}\right)-F_{0}(\phi)\right)-D F(\phi)(\psi)\right| \\
& \quad+\int_{0}^{\infty} \int_{0}^{x} e^{-\frac{x-s}{\lambda}}\left|t^{-1}\left(G_{0}\left(\phi_{t}\right)(s)-G_{0}(\phi)(s)\right)-D G(\phi)(\psi)(s)\right| d s d x \\
& \leq \lambda\left|t^{-1}\left(F_{0}\left(\phi_{t}\right)-F_{0}(\phi+t \psi)\right)+\lambda\right| t^{-1}\left(F_{0}(\phi+t \psi)-F_{0}(\phi)\right)-D F(\phi)(\psi) \mid \\
& \quad+\lambda\left\|t^{-1}\left(G_{0}\left(\phi_{t}\right)-G_{0}(\phi+t \psi)\right)\right\|_{L^{1}} \\
& \quad+\lambda\left\|t^{-1}\left(G_{0}(\phi+t \psi)-G_{0}(\phi)\right)-D G(\phi)(\psi)\right\|_{L^{1}} \\
& \leq \lambda\left\|F_{0}\right\|_{\text {Lip }}\left\|t^{-1}\left(\phi_{t}-\phi\right)-\psi\right\|_{L^{1}}+\lambda\left|t^{-1}\left(F_{0}(\phi+t \psi)-F_{0}(\phi)\right)-D F(\phi)(\psi)\right| \\
& \quad+\lambda\left\|G_{0}\right\|_{\text {Lip }}\left\|t^{-1}\left(\phi_{t}-\phi\right)-\psi\right\|_{L^{1}}+\lambda\left\|t^{-1}\left(G_{0}(\phi+t \psi)-G_{0}(\phi)\right)-D G(\phi)(\psi)\right\|_{L^{1}} .
\end{aligned}
$$

Hence we have $(1-\lambda \omega)\left\|t^{-1}\left(\phi_{t}-\phi\right)-\psi\right\|_{L^{1}} \leq \lambda\left(\left|t^{-1} o_{F}(t \psi)\right|+\left\|t^{-1} o_{G}(t \psi)\right\|_{L^{1}}\right)$ for sufficiently small $t>0$. This leads to the consequence.

The next proposition shows that $\partial A(u)$ is the proto-derivative of $A$ at $u$. Proposition 5.4. For $u \in \mathscr{D}(A) \cap B_{r}(\bar{u})$,

$$
\mathscr{G}(\partial A(u))=\lim _{t \downarrow 0} t^{-1}[\mathscr{G}(A)-(u, A u)] .
$$

Proof. Let $v=(I+\lambda A) u$ for $u \in \mathscr{D}(A) \cap B_{r}(\bar{u})$ and $0<\lambda<1 / 2 \omega$. By Lemma 5.3, $d J_{\lambda}(v) h=J_{\lambda}^{\partial A\left(J_{\lambda} v\right)} h$. Define $\Psi_{\lambda}(x, y)=(x+\lambda y, x)$. Then by [9, Lemma 4.1], we obtain

$$
\begin{aligned}
\lim _{t \downarrow 0} t^{-1}\left[\Psi_{\lambda}^{-1}\left(\mathscr{G}\left(J_{\lambda}\right)\right)-\Psi_{\lambda}^{-1}\left(v, J_{\lambda} v\right)\right] \\
=\Psi_{\lambda}^{-1}\left(\mathscr{G}\left(d J_{\lambda}(v)\right)\right)=\Psi_{\lambda}^{-1}\left(\mathscr{G}\left(J_{\lambda}^{\partial A\left(J_{\lambda} v\right)}\right)\right) .
\end{aligned}
$$

Since $\Psi_{\lambda}\left(\mathscr{G}\left(J_{\lambda}^{Q}\right)\right)=\mathscr{G}(Q)$, the above reads $\lim _{t \downarrow 0} t^{-1}\left[\mathscr{G}(A)-\left(J_{\lambda} v, A J_{\lambda} v\right)\right]=$ $\mathscr{G}\left(\partial A\left(J_{\lambda} v\right)\right)$, which is the result.

Proposition 5.5. $\partial A(u)+\omega I$ is $m$-accretive in $L^{1}$ for $u \in \mathscr{D}(A) \cap B_{r}(\bar{u})$.

Proof. From Propositions 5.2 and 5.4, this is shown in a similar way to Corollary 4.4 and so we omit the details.

Finally, we have

Proposition 5.6. There exist $\lambda_{\bar{u}}>0, \delta_{\bar{u}}>0$, and a nondecreasing $L_{\bar{u}}:[0, \infty) \rightarrow$ $[0, \infty)$ such that

$$
\left\|J_{\lambda}^{\partial A(z)} v-J_{\lambda}^{\partial A(u)} v\right\|_{L^{1}} \leq \lambda\|z-u\|_{L^{1}} L_{\bar{u}}\left(\|v\|_{L^{1}}\right)
$$

for $0<\lambda<\lambda_{\bar{u}}, z, u \in B_{\delta_{u}}(\bar{u}) \cap \mathscr{D}(A)$ and $v \in L^{1}$.

Proof. Let $z, u \in B_{r}(\bar{u}) \cap \mathscr{D}(A), v \in L^{1}$ and put $\psi=J_{\lambda}^{\partial A(z)} v, \phi=J_{\lambda}^{\partial A(u)} v$. Then these satisfy

$$
\begin{aligned}
& \psi(x)=e^{-\frac{x}{\lambda}} D F(z)(\psi)+\int_{0}^{x} e^{-\frac{x-s}{\lambda}}\left[\frac{1}{\lambda} v(s)+D G(z)(\psi)(s)\right] d s, \\
& \phi(x)=e^{-\frac{x}{\lambda}} D F(u)(\phi)+\int_{0}^{x} e^{-\frac{x-s}{\lambda}}\left[\frac{1}{\lambda} v(s)+D G(u)(\phi)(s)\right] d s .
\end{aligned}
$$


Hence we have the estimate

$$
\begin{aligned}
\|\psi-\phi\|_{L^{1}} \leq & \int_{0}^{\infty} e^{-\frac{x}{\lambda}} d x|D F(z)(\psi)-D F(u)(\phi)| \\
& +\int_{0}^{\infty} \int_{0}^{x} e^{-\frac{x-s}{\lambda}}|D G(z)(\psi)(s)-D G(u)(\phi)(s)| d s d x \\
\leq & \lambda(|D F(z)(\psi)-D F(u)(\psi)|+|D F(u)(\psi)-D F(u)(\phi)|) \\
& +\lambda\left(\|D G(z)(\psi)-D G(u)(\psi)\|_{L^{1}}+\|D G(u)(\psi)-D G(u)(\phi)\|_{L^{1}}\right) \\
\leq & \lambda|D F(z)(\psi)-D F(u)(\psi)|+\lambda\|D F(u)\|_{\mathscr{L}\left(L^{1}, E\right)}\|\psi-\phi\|_{L^{1}} \\
& +\lambda\|D G(z)(\psi)-D G(u)(\psi)\|_{L^{1}}+\lambda\|D G(u)\|_{\mathscr{L}\left(L^{1}, L^{1}\right)}\|\psi-\phi\|_{L^{1}} .
\end{aligned}
$$

By the continuity of $u \mapsto\|D F(u)\|_{\mathscr{L}\left(L^{1}, E\right)},\|D G(u)\|_{\mathscr{L}\left(L^{1}, L^{1}\right)}$, there exists a $\delta>$ 0 such that $\|u-\bar{u}\|_{L^{1}}<\delta$ implies $\|D F(u)\|_{\mathscr{L}\left(L^{1}, E\right)} \leq 1 / 2+\|D F(\bar{u})\|_{\mathscr{L}\left(L^{1}, E\right)}$ and $\|D G(u)\|_{\mathscr{L}\left(L^{1}, L^{1}\right)} \leq 1 / 2+\|D G(\bar{u})\|_{\mathscr{L}\left(L^{1}, L^{1}\right)}$. Further, there exists a $\lambda_{\bar{u}}>0$ such that $0<\lambda<\lambda_{\bar{u}}$ implies that $\lambda\left(1+\|D F(\bar{u})\|_{\mathscr{L}\left(L^{1}, E\right)}+\|D G(\bar{u})\|_{\mathscr{L}\left(L^{1}, L^{1}\right)}\right) \leq 1 / 2$ and $1-\lambda \omega \geq 1 / 2$. Therefore, from the above estimate, it follows that

$$
\|\psi-\phi\|_{L^{1}} \leq 2 \lambda\left(|D F(z)(\psi)-D F(u)(\psi)|+\|D G(z)(\psi)-D G(u)(\psi)\|_{L^{1}}\right) .
$$

Since $z, u \in B_{r}(\bar{u})$ implies $z, u \in B_{r_{0}}(0)$, one has by $(\mathrm{F})$ and $(\mathrm{G})$,

$$
\begin{aligned}
|D F(z)(\psi)-D F(u)(\psi)| & \leq d_{F}\left(r_{0}\right)\|z-u\|_{L^{1}}\|\psi\|_{L^{1}} \leq 2 d_{F}\left(r_{0}\right)\|z-u\|_{L^{1}}\|v\|_{L^{1}}, \\
\|D G(z)(\psi)-D G(u)(\psi)\|_{L^{1}} & \leq d_{G}\left(r_{0}\right)\|z-u\|_{L^{1}}\|\psi\|_{L^{1}} \leq 2 d_{G}\left(r_{0}\right)\|z-u\|_{L^{1}}\|v\|_{L^{1}} .
\end{aligned}
$$

Putting $\delta_{\bar{u}}:=\min \{\delta, r\}>0$ and $L_{\bar{u}}(s):=4\left(d_{F}\left(r_{0}\right)+d_{G}\left(r_{0}\right)\right) s$, we reach the desired inequality.

Consequently, the hypotheses (H1) and (H2) are fulfilled. Let $\{S(t)\}$ be a nonlinear semigroup generated by $-A$. Then $u(t):=S(t) u_{0}$ is regarded as a generalized solution of (P) with $F$ and $G$ replaced by $F_{0}$ and $G_{0}$ and with initial data $u_{0}$. If $u(t)$ lies in the ball $B_{r_{0}}(0)$, then we may think of $u(t)$ as a generalized solution of the original problem $(\mathrm{P})$ since $F_{0}$ and $G_{0}$ are identical to $F$ and $G$ on $B_{r_{0}}(0)$, respectively. Therefore we have the following conclusion similar to [17].

Theorem 5.7. If $\omega_{0}(-\partial A(\bar{u}))<0$, then $\bar{u}$ is exponentially asymptotically stable in the sense that there exist constants $\eta>0, M \geq 1$ and $\alpha>0$ such that if $\left|u_{0}-\bar{u}\right|<\eta$, then the solution $u(t)$ of $(P)$ with initial data $u_{0}$ in the generalized sense described above exists uniquely for all $t \geq 0$ and satisfies $|u(t)-\bar{u}| \leq M e^{-\alpha t}\left|u_{0}-\bar{u}\right|$ for all $t \geq 0$.

\section{SEMILINEAR FUNCTIONAL DIFFERENTIAL EQUATIONS}

In this section, we discuss briefly the application to semilinear functional differential equations. Let $(E,|\cdot|)$ be a Banach space, $L: \mathscr{D}(L) \subset E \rightarrow E$ be a linear operator such that $-L+\beta I$ is $m$-accretive in $E$, where $\beta \in \mathbb{R}$. Let $C:=$ $C([-r, 0] ; E)$ be a Banach space with norm $\|\phi\|_{C}:=\sup _{\theta \in[-r, 0]}|\phi(\theta)|$ and $F: C \rightarrow E$ be a nonlinear operator which is continuously Fréchet differentiable on $C$ as defined in $\S 1$. In this section, we consider the semilinear functional differential equation

$$
(d / d t) x(t)=L x(t)+F\left(x_{t}\right), \quad t \geq 0,
$$


where $x_{t}(\theta)=x(t+\theta), \theta \in[-r, 0]$. We say that $\bar{x}$ is a stationary solution of (FDE) if $\bar{x} \in \mathscr{D}(L)$ and $L \bar{x}+F(\bar{x})=0$, where $\bar{x}$ is considered as a constant function in $C$. Let the stationary solution $\bar{x}$ of (FDE) be fixed and take $r_{0}>0$ such that $\|\bar{x}\|_{C}<r_{0}$. Let $F_{0}$ be the radial truncation of $F$ defined similarly in $\S 5$ (see also Remark 1.1) and define an operator $A$ on $C$ by

$$
\begin{aligned}
\mathscr{D}(A) & =\left\{f \in C \mid f^{\prime} \in C, f(0) \in \mathscr{D}(L), f^{\prime}(0)=L f(0)+F_{0}(f)\right\}, \\
A f & =-f^{\prime} \text { for } f \in \mathscr{D}(A) .
\end{aligned}
$$

Then as shown in [18], $A+\omega I, \omega=\max \left\{0, \beta+\left\|F_{0}\right\|_{\text {Lip }}\right\}$ is $m$-accretive in $C$ and densely defined. Similarly to $\S 4$, we can show that for sufficiently small $r>0$, the conditions (H1) and (H2) in $\S 2$ are fulfilled for $A$ with the proto-derivative $\partial A(x)$ at $x \in \mathscr{D}(A) \cap B_{r}(\bar{x})$ defined by

$$
\begin{aligned}
\mathscr{D}(\partial A(x)) & =\left\{h \in C \mid h^{\prime} \in C, h(0) \in \mathscr{D}(L), h^{\prime}(0)=L h(0)+D F(x)(h)\right\} \\
\partial A(x) h & =-h^{\prime} \quad \text { for } h \in \mathscr{D}(\partial A(x)),
\end{aligned}
$$

where $B_{r}(\bar{x})$ is a ball in $C$. Denoting by $\{S(t)\}$ a semigroup on $C$ generated by $-A$, the function $x(t)$ defined by

$$
x(t)= \begin{cases}\phi(t) & \text { for }-r \leq t \leq 0, \\ {[S(t) \phi](0)} & \text { for } t>0\end{cases}
$$

for $\phi \in C$, is considered as a generalized solution of (FDE) with $F$ replaced by $F_{0}$ and with initial history $\phi$. For sufficient conditions for $x(t)$ to be a classical solution, see [18]. If $x(t)$ belongs to the ball $B_{r_{0}}(0)$, then we may regard $x(t)$ as a generalized solution of the original problem (FDE). By applying Theorem 2.1, we have the following result similar to [11].

Theorem 6.1. Let $\bar{x}$ be a stationary solution of (FDE). If $\omega_{0}(-\partial A(\bar{x}))<0$, then $\bar{x}$ is exponentially asymptotically stable, i.e., there exist constants $\eta>0$, $M \geq 1, \alpha>0$ such that

$$
\left\|x_{t}-\bar{x}\right\|_{C} \leq M e^{-\alpha t}\|\phi-\bar{x}\|_{C}, \quad t \geq 0,
$$

whenever $\phi \in C$ and $\|\phi-\bar{x}\|_{C}<\eta$.

Remark 6.1. In particular, applying Plant's results [12, Corollary 3.2], we can obtain that if $\beta+\|D F(\bar{x})\|<0$, then $\omega_{0}(-\partial A(\bar{x}))<0$. Indeed, [12, Corollary 3.2] tells that there exists a $w<0$ for which $\partial A(\bar{u})+w I$ is $m$-accretive in $C$ with the weighted norm $|\phi|_{w}=\sup _{\theta \in[-r, 0]}\left|e^{-w \theta} \phi(\theta)\right|$. Since the norm $|\cdot|_{w}$ is equivalent to the original norm $\|\cdot\|_{C}$, it is shown that $-\partial A(\bar{u})$ is an infinitesimal generator of a $\left(C_{0}\right)$-semigroup $\{T(t)\}$ on $C$ such that $\|T(t) \phi\|_{C} \leq$ $M e^{w t}\|\phi\|_{C}$ for some $M \geq 1$.

\section{REFERENCES}

1. J. P. Aubin and I. Ekeland, Applied nonlinear analysis, Wiley-Interscience, New York, 1984.

2. V. Barbu, Nonlinear semigroups and differential equations in Banach spaces, Noordhoff, Groningen, 1976.

3. H. Brezis, Opérateurs maximaux monotones et semigroupes de contractions dans les espace de Hilbert, North-Holland, Amsterdam, 1973.

4. W. Desch and W. Schappacher, Linearized stability for nonlinear semigroups, Differential Equations in Banach Spaces (A. Favini and E. Obrecht, eds.), Lecture Notes in Math., vol. 1223, Springer-Verlag, 1986, pp. 61-73. 
5. H. Frankowska, Local controllability and infinitesimal generators of semigroups of set-valued maps, SIAM J. Control 25 (1987), 412-432.

6. A. Grabosch, Translation semigroups and their linearizations on spaces of integrable functions, Trans. Amer. Math. Soc. 311 (1989), 357-390.

7. G. Greiner, Linearized stability for hyperbolic evolution equations with semilinear boundary conditions, Semigroup Forum 38 (1989), 203-214.

8. D. Henry, Geometric theory of semilinear parabolic equations, Lecture Notes in Math., vol. 840, Springer-Verlag, 1981.

9. N. Kato, On the second derivatives of convex functions on Hilbert spaces, Proc. Amer. Math. Soc. 106 (1989), 697-705.

10. S. Oharu and T. Takahashi, Characterization of nonlinear semigroups associated with semilinear evolution equations, Trans. Amer. Math. Soc. 311 (1989), 593-619.

11. M. E. Parrot, Positivity and a principle of linearized stability for delay-differential equations, Differential Integral Equations 2 (1989), 170-182.

12. A. T. Plant, Stability of nonlinear functional differential equations using weighted norms, Houston J. Math. 3 (1977), 99-108.

13. J. Prüß, Stability analysis for equibria in age-specific population dynamics, Nonlinear Anal. 7 (1983), 1291-1312.

14. R. T. Rockafellar, Proto-differentiability of set-valued mappings and its applications in optimization, Ann. Inst. Poincaré Analyse Non Linéaire 6 (1989), 449-482.

15. N. Sanekata, Abstract quasi-linear equations of evolution in nonreflexive Banach spaces, Hiroshima Math. J. 19 (1989), 109-139.

16. J. Smoller, Shock waves and reaction-diffusion equations, Springer-Verlag, 1982.

17. G. F. Webb, Theory of nonlinear age-dependent population dynamics, Marcel Dekker, New York, 1985.

18. G. F. Webb, Asymptotic stability for abstract nonlinear functional differential equations, Proc. Amer. Math. Soc. 54 (1976), 225-230.

19. G. F. Webb, Continuous nonilinear perturbations of linear accretive operators in Banach spaces, J. Funct. Anal. 10 (1972), 191-203.

Department of Mathematics, Shimane University, Matsue 690, Japan

E-mail address: nkato@shimane-u.ac.jp 